\title{
Virtual reality mobile application to improve videoscopic airway training: A randomised trial
}

Ying Wei $\underline{\text { Yau }},{ }^{1,2}{ }_{M C I}$, Zisheng $\underline{\operatorname{Li}},{ }^{1,2}{ }_{\text {MMed (EM) }}$, Mui Teng $\underline{\text { Chua }},{ }^{1,2}{ }_{M P H}$, Win Sen $\underline{\text { Kuan, }},{ }^{1,2}{ }_{M C I}$, Gene Wai Han $\underline{\text { Chan }},{ }^{1,2}{ }_{M M e d(E M)}$

\begin{abstract}
Introduction: Flexible bronchoscopic intubation (FBI) is an important technique in managing an anticipated difficult airway, yet it is rarely performed and has a steep learning curve. We aim to evaluate if the integration of virtual reality gaming application into routine FBI training for emergency department doctors would be more effective than traditional teaching methods.

Methods: We conducted a randomised controlled trial to compare self-directed learning using the mobile application, Airway Ex* in the intervention group versus the control group without use of the mobile application. All participants underwent conventional didactic teaching and low-fidelity simulation with trainer's demonstration and hands-on practice on a manikin for FBI. Participants randomised to the intervention arm received an additional 30 minutes of self-directed learning using Airway Ex, preloaded on electronic devices while the control arm did not. The primary outcome was time taken to successful intubation.

Results: Forty-five physicians (20 junior and 25 senior physicians) were enrolled, with male predominance $(57.8 \%, 26 / 45)$. There was no difference in time taken to successful intubation (median 48 seconds [interquartile range, IQR 41-69] versus 44 seconds [IQR 37-60], $P=0.23$ ) between the control and intervention groups, respectively. However, the intervention group received better ratings (median 4 [IQR 4-5]) for the quality of scope manipulation skills compared to control (median 4 [IQR 3-4], adjusted $P=0.03$ ). This difference remains significant among junior physicians in stratified analysis.

Conclusion: Incorporating virtual reality with traditional teaching methods allows learners to be trained on FBI safely without compromising patient care. Junior physicians appear to benefit more compared to senior physicians.
\end{abstract}

Ann Acad Med Singap 2021;50:141-8

Keywords: Airway management, emergency medicine, intubation, simulation education, virtual reality

\section{INTRODUCTION}

Emergency airway management is a keystone of emergency medicine practice and critical skill in residency training. An accredited emergency attending is expected to handle difficult airways that may present unexpectedly with expertise. Flexible bronchoscopic intubation (FBI) technique is considered an important option in the management of predicted difficult airways. In such situations, awake intubation with FBI is the preferred choice among anaesthesiologists. ${ }^{1}$ However, it is rarely performed in daily practice in the emergency department $(\mathrm{ED})^{2}$ and yet emergency physicians are expected to be adept at performing this skill in a crisis scenario. For instance, in patients with severe airway burns where routine orotracheal intubation is expected to be difficult, FBI may be a more ideal first-line technique to secure the airway since it allows for preintubation evaluation of the anatomy followed by bronchoscopic-guided intubation. ${ }^{3}$ Apart from the lack of clinical practice, there is a steep learning curve to master this complex psychomotor skill. ${ }^{4}$

Providing sufficient training in FBI, particularly hands-on experience with patients is also not without its challenges. Patients with known difficult airway

\footnotetext{
${ }^{1}$ Emergency Medicine Department, National University Hospital, National University Health System, Singapore

${ }^{2}$ Department of Surgery, Yong Loo Lin School of Medicine, National University of Singapore, Singapore

Correspondence: Dr Mui Teng Chua, Emergency Medicine Department Office, National University Hospital, 9 Lower Kent Ridge Road, Level 4, Singapore 119085. Email: mui_teng_chua@nuhs.edu.sg

*Feature of the gaming application in this article is not part of an advertised endorsement. User discretion in applying the gaming application to clinical practice is advised.
} 


\section{CLINICAL IMPACT}

\section{What is New}

- Integration of virtual reality gaming application into conventional didactic training for flexible bronchoscopic intubation for added self-directed learning, anytime and anywhere.

- Gaming applications that continuously present evolving clinical scenarios can provide diverse and unpredictable practice tasks.

\section{Clinical Implications}

- Training using virtual reality, together with traditional teaching methods will allow learners to train and practise in a safe environment without compromising patient care.

- Apart from teaching, virtual reality simulations may be useful for competency assessment, which is only possible opportunistically in reality.

requiring FBI infrequently present to the ED. ${ }^{5}$ Unlike patients presenting for elective surgeries with mostly normal airways, the unpredictable airway anatomy and urgent interventions in the ED render real-life FBI training impossible in this setting. To avoid technical and ethical concerns of training involving patients, ${ }^{6}$ conventional teaching methods incorporate the use of a low-fidelity manikin. ${ }^{7}$ However, the manikin anatomy often lacks the realism of a live human. ${ }^{8}$ The addition of virtual reality technology, in the form of a low-cost mobile gaming application (app), Airway Ex (Level Ex, Chicago, US) into conventional simulation, may optimise learning by providing an ethical, cost-effective and more realistic modality to acquire the basic skills of FBI. ${ }^{9}$ The aim of our study is to test the hypothesis that integrating virtual reality via a gaming app into conventional didactic and hands-on training of FBI for ED physicians and residents would be more effective than traditional teaching methods alone.

\section{METHODS}

\section{Trial design and oversight}

The Assessing Usefulness of virtual Reality mobile app in flexible bronchoscope Airway training (AURA) study is a randomised controlled trial conducted at the ED of the National University Hospital in Singapore, a member of the National University Health System.
The trial protocol (Fig. 1) was approved and granted waiver of documentation of consent by the National Healthcare Group, Domain Specific Review Board (DSRB reference number: 2018/00554) and registered on ClinicalTrials.gov (Identifier: NCT03663296).

\section{Participants}

Participants were stratified into 2 groups: junior and senior physicians. Junior physicians included junior residents, medical officers and resident physicians, while senior physicians comprised those who have obtained an Emergency Medicine diploma or Master of Medicine certification or equivalent in Singapore, and who routinely undertook supervisory roles to the junior physicians during clinical shifts. All doctors, with varying prior intubation and airway management experience, from our ED were invited to participate.

\section{Randomisation and blinding}

Participants were randomised in a 1:1 ratio to the control or intervention group, stratified by seniority (junior versus senior physicians). Block-of-4 randomisation sequence was generated using a web-based randomisation tool (https://www.sealedenvelope.com/simple-randomiser/ $\mathrm{v} 1 /$ lists) for each stratum. The allocation was concealed within sealed opaque envelopes, and selected consecutively. Once the participants consented to enter the study, the envelope was opened, and they were informed of the allocation. Only the primary investigator not involved in the training or assessment was aware of the allocation. Both trainers and assessors were blinded to the allocation to ensure similar quality of teaching delivered and outcome assessment for both arms.

\section{Trial intervention}

All participants underwent conventional didactic teaching and low-fidelity simulation with trainer's demonstration and hands-on practice on a manikin using the flexible bronchoscope attached to a C-MAC monitor (Karl Storz SE \& Co. KG, Tuttlingen, Germany) for a total of 30 minutes. Thereafter, participants who were randomised to the intervention arm received an additional 30 minutes of self-directed learning using the mobile app (Airway Ex), preloaded into study electronic devices. Those in the control group were asked to remain in the same private enclosed room during this period without any practice with the app.

Airway Ex is a mobile gaming app that allows users to practise virtual intubations with realistic visuals and controls using their mobile phones or tablet devices (Fig. 2). To ensure that the intervention group participants 


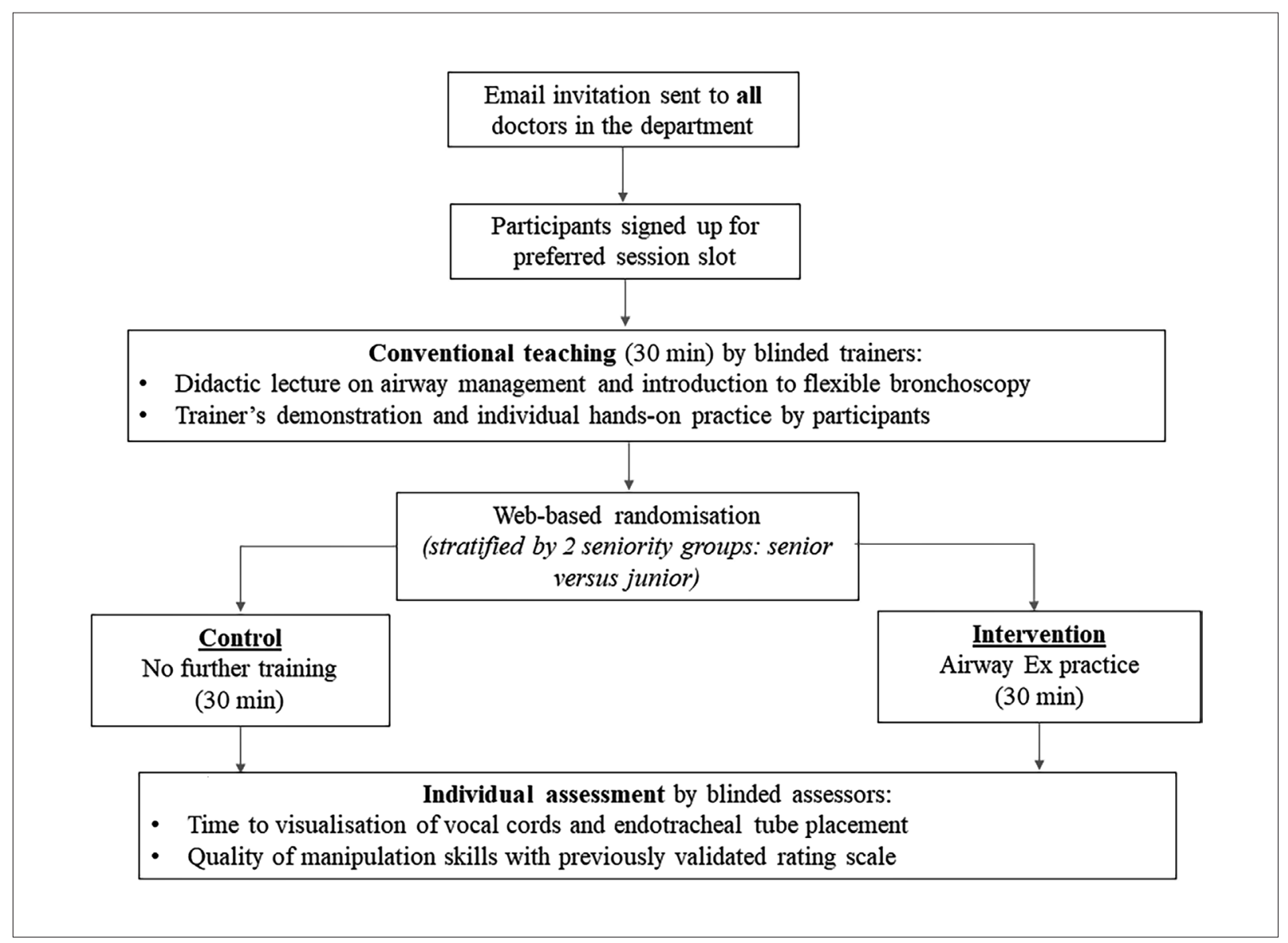

Fig. 1. The AURA trial protocol.

AURA: Assessing Usefulness of virtual Reality mobile application in flexible bronchoscope Airway training

remain actively engaged in the learning process, they were given a set of instructions to attempt 6 different cases using the app, deliberately arranged in increasing levels of difficulty. The 6 cases pre-selected for the intervention group training consisted of:

1. Unit 0 -Airway Ex tutorial (introduction to the mobile app interface and manipulation of the virtual scope)

2. Unit 0 -Tutorial: intubation with flexible scope (provides real-time feedback on scope manipulation skills)

3. Unit 1-Adult intubation: glottic tumour

4. Unit 2 - Rare supraglottic tumour

5. Unit 2 - Upright awake intubation

6. Unit 2 - Case challenge: epiglottitis

To enhance the level of engagement of participants, they were also asked to record the highest score that they have attained for Unit 1 . They could reattempt the same case or practise other cases if they had completed the 6 cases before their allotted 30-minute of selfpractice elapsed.
Each participant was then individually assessed in a private enclosed room. They were asked to perform FBI using the same manikin and equipment that they had used during their practice session with the trainer earlier. The first assessor (ZL) recorded the timings to visualisation of vocal cords and successful intubation using an electronic stopwatch while the second assessor (GWHC) observed and rated the quality of manipulation skills for all the participants. Additionally, all participants were also asked to complete a questionnaire to rate their confidence levels.

\section{Outcomes}

The primary outcome was time taken to visualisation of the vocal cords and endotracheal tube placement (i.e. successful intubation) on the manikin. The attempt was considered as failed if the endotracheal tube was not passed through the cords within a pre-determined time period of 120 seconds. Other outcomes of interest include proportion of successful intubation; quality of scope manipulation skills as assessed by a validated 5-point 

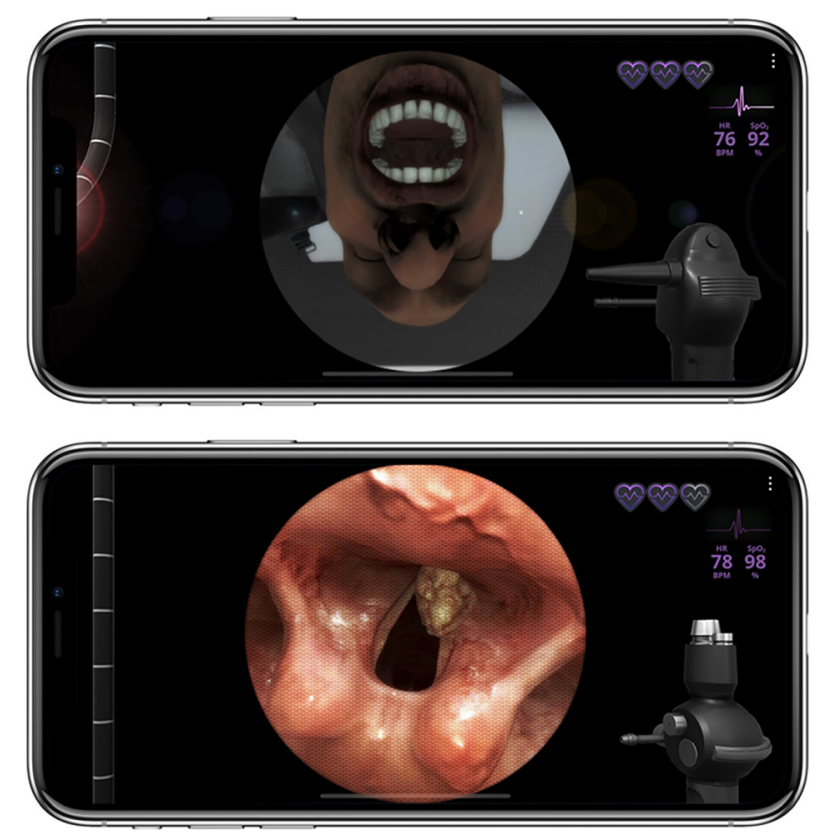

Fig. 2. Images of a virtual patient with a glottic tumour on Airway Ex. Source: Airway Ex application. Reproduced with permission from Level Ex Inc., Chicago, US.

global rating scale for flexible bronchoscope manipulation ability ${ }^{5}$ and self-rated confidence scores in performing FBI on a real patient before and after the training using a Likert scale from 0 to 10 (0 being with no/little confidence to 10 as extremely confident).

\section{Statistical analysis}

The sample size was calculated to detect at least a 20 -second time difference ( $\mathrm{SD} \pm 15$ seconds) in successful FBI between the control and intervention group in each stratum, with $80 \%$ power and a two-tailed alpha of $5 \%$. The time difference of 20 seconds was chosen as critically ill patients may desaturate in such a short time-span during apnoea. ${ }^{10}$ A minimum sample size of 20 participants (10 in each arm) was required. To allow for comparison and analysis within the junior and senior strata, we aimed to enrol at least 20 junior and 20 senior physicians.

The time taken for visualisation of the vocal cords and successful intubation, proportion of successful intubation, quality of manipulation skills, and participant's confidence scores were compared between the intervention and control groups, and within each stratum (i.e. junior and senior physician groups). All data were populated in a Microsoft Excel spreadsheet (Microsoft Corp, Redmond, US). Upon completion of data collection electronically, the charts were reviewed for missing or duplicated data, and verified. The data were then exported into Stata 15 (StataCorp LP, College Station, US) for statistical analyses. Categorical variables are reported as frequency and percentage values. Parametric continuous variables are reported as mean (standard deviation [SD]) and analysed using Student's t-test. Non-parametric continuous variables are reported as median (interquartile range $[\mathrm{IQR}]$ ) and analysed using Mann-Whitney $U$ test. Statistical significance was set at $P<0.05$.

\section{RESULTS}

\section{Study population and characteristics}

Forty-five physicians (20 junior and 25 senior physicians) were enrolled between 24 July 2018 and 11 August 2018 (Fig. 3). Three physicians, who attended the training session, declined participation in the study and were excluded. There was no loss to follow-up.

The characteristics of the control and intervention groups are shown in Table 1. There were more male physicians in the intervention group $(72.7 \%$ versus $43.4 \%$ ). Two participants in the control group had prior FBI experience while none in the intervention group had ever performed an FBI.

\section{Outcomes}

Between the control and intervention groups, there were no significant differences in time taken to visualise the vocal cords (median 13 seconds [IQR 9-38] versus 12 seconds [IQR 8-22], $P=0.36$ ) and to successful intubation (median 48 seconds [IQR 41-69] versus 44 seconds [IQR 37-60], $P=0.23$ ), outlined in Table 2.

Two participants in the control group $(8.7 \%)$ failed to place the endotracheal tube correctly within 120 seconds whereas there were no failures in the intervention group (Table 2). The intervention group also received better ratings (median 4 [IQR 4-5]) for the quality of scope manipulation skills compared to the control group (median 4 [IQR 3-4]). This difference in skill quality remained statistically significant even after adjusting for age, gender, physician rank and previous FBI experience (crude $P=0.04$, adjusted $P=0.03$ ). There were no differences in the change in participant's confidence scores between the control and intervention groups $(P=0.80)$ (Table 2$)$.

\section{Pre-specified strata analysis}

Within the intervention arm, there was no statistically significant results between the junior and senior strata in terms of time taken to visualise vocal cords $(P=0.209)$ and successful intubation $(P=0.176)$, and scores for scope manipulation skills $(P=0.221)$ (Table 3$)$. For the control arm, there were no significant differences in the timings between the senior and junior physicians 


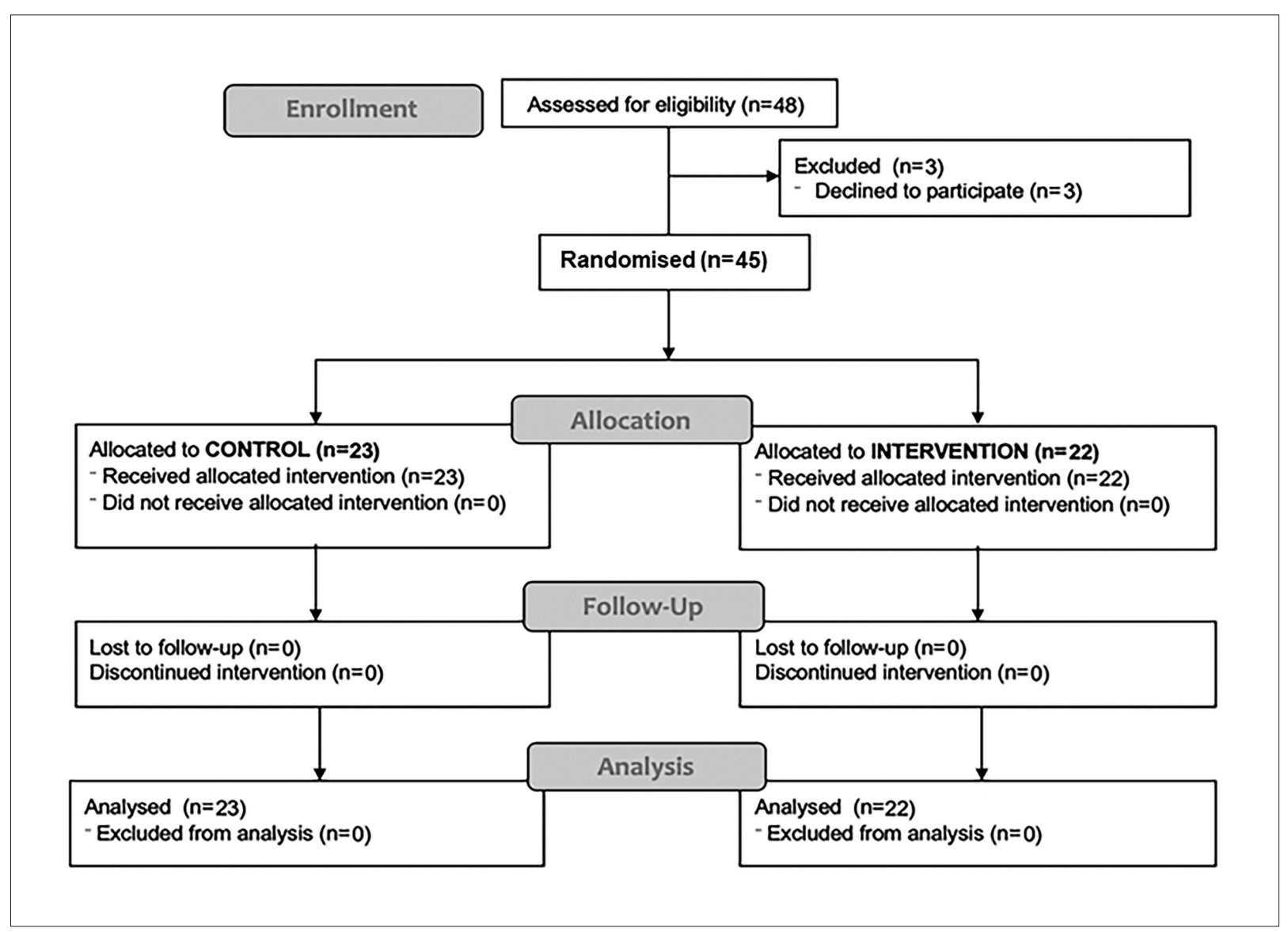

Fig. 3. The CONSORT flow diagram of the randomised trial of the 2 groups.

Source: The CONSORT (2010) flow diagram. Available at: http://www.consort-statement.org/consort-statement/flow-diagram.

Table 1. Baseline characteristics of participants $(\mathrm{N}=45)$

\begin{tabular}{|c|c|c|}
\hline Variables & $\begin{array}{l}\text { Control n }(\%) \\
\qquad(n=23)\end{array}$ & $\begin{array}{l}\text { Intervention } n(\%) \\
\qquad(\mathrm{n}=22)\end{array}$ \\
\hline Age in years, median (IQR) & $29(27-38)$ & $30(28-41)$ \\
\hline Male gender & $10(43.4)$ & $16(72.7)$ \\
\hline Post-graduate years of experience, median (IQR) & $5(4-14)$ & $6(4-14)$ \\
\hline Previous FBI experience ${ }^{\mathrm{b}}$ & $2(8.6)$ & $0(0)$ \\
\hline
\end{tabular}

FBI: flexible bronchoscopic intubation; IQR: interquartile range

a Senior physicians in our department comprise resident physicians who have obtained an Emergency Medicine diploma, Master of Medicine certification or equivalent in Singapore, with supervisory roles; senior residents; and Emergency Medicine (EM) attendings (board-certified EM specialists).

${ }^{\mathrm{b}}$ Previous FBI experience in clinical settings; none of the participants has undergone formal FBI training.

( $P=0.804$ for vocal cords visualisation; $P=0.828$ for successful intubation). However, the senior physicians in the control group achieved higher scope manipulation ratings compared to the junior physicians $(P=0.021)$.

Among the junior physicians, there was no difference in time taken for visualisation of the vocal cords $(P=0.97)$ between intervention and control groups
(Table 3). However, the intervention group took a much shorter time (median improvement of 11 seconds [IQR -5-27 seconds]) to successful intubation, although this result did not reach statistical significance $(P=0.12)$. There were no failures observed. The intervention group in the junior physician strata also received higher ratings for manipulation skill quality compared to the control 
Table 2. Results of primary outcomes

\begin{tabular}{lccc}
\hline Primary Outcomes & Control (n=23) & Intervention (n=22) & P value \\
\hline $\begin{array}{l}\text { Time taken (in seconds) to } \\
\text { visualise vocal cords }\end{array}$ & $13(9-38)$ & $44(37-60)$ & 0.36 \\
$\begin{array}{l}\text { Time taken (in seconds) to } \\
\text { successful intubation }\end{array}$ & $48(41-69)$ & 0 & 0.23 \\
$\begin{array}{l}\text { Failure to achieve tube placement within } 120 \text { seconds } \\
\text { Rating scores for quality of } \\
\text { scope manipulation skills (1 to 5) }\end{array}$ & $2(8.7)$ & $4(4-5)$ & NA \\
\hline $\begin{array}{l}\text { Self-rated improvement in } \\
\text { confidence scores (0 to 10) }\end{array}$ & $4(3-4)$ & $3.5(2-5)$ & 0.04 \\
\hline
\end{tabular}

All values stated as medians (interquartile range), unless otherwise stated.

(median 4 [IQR 4-4] versus 3 [IQR 3-4], crude $P=0.006)$. This difference remained statistically significant after adjusting for differences in age, gender and previous experience with FBI between the two groups (adjusted $P=0.02$ ).

Within the senior physician strata, there were 2 failures seen in the control group. There were no differences in the time taken to visualise vocal cords and successful endotracheal tube placement, scope manipulation skill quality between the control and intervention groups (Table 3).

\section{DISCUSSION}

The application of simulation and virtual reality in education is well documented in aerospace and astronaut training. ${ }^{11}$ It has played a ubiquitous role in exposing learners to skills and situations that are not commonly encountered in reality and yet are essential to master in crisis scenarios. With advancement in technology, medical education has evolved in the past 2 decades and shifted away from an apprenticeship model to incorporation of simulation and virtual reality. ${ }^{12}$

Indeed, the use of virtual reality can be advantageous to medical education. The system of having novices practising procedures on real-life patients has always been controversial, leading to ethical issues concerning patient safety and quality of care. The use of virtual reality offers learners and educators an environment devoid of such risks to practise decision-making and procedures, allowing them to experience scenarios that mirror real-life situations. ${ }^{13}$ The ability to generate and continuously present evolving clinical scenarios in

Table 3. Pre-specified subgroup results comparing junior and senior physicians' performance

\begin{tabular}{|c|c|c|c|}
\hline Outcomes & Control & Intervention & $P$ value \\
\hline \multicolumn{4}{|c|}{ Time taken (in seconds) to visualise vocal cords ${ }^{\mathrm{a}}$} \\
\hline Junior $(\mathrm{n}=20)$ & $13(9-24)$ & $13.5(10-22)$ & 0.97 \\
\hline Senior $(n=25)$ & $12(10-38)$ & $8.5(6.5-23)$ & 0.23 \\
\hline \multicolumn{4}{|c|}{ Time taken (in seconds) to successful intubation ${ }^{\mathrm{b}}$} \\
\hline Junior $(\mathrm{n}=20)$ & $49.5(43-62)$ & $38.5(33-53)$ & 0.12 \\
\hline Senior $(\mathrm{n}=25)$ & $48(41-69)$ & $45.5(40.5-61.5)$ & 0.87 \\
\hline \multicolumn{4}{|c|}{ Failure to achieve tube placement within 120 seconds, $n$ (\%) } \\
\hline Junior $(n=20)$ & 0 & 0 & NA \\
\hline Senior $(n=25)$ & $2(15.4)$ & 0 & \\
\hline \multicolumn{4}{|c|}{ Rating scores for quality of scope manipulation skills ${ }^{\mathrm{c}}$} \\
\hline Junior $(\mathrm{n}=20)$ & $3(3-4)$ & $4(4-4)$ & 0.006 \\
\hline Senior $(n=25)$ & $4(4-5)$ & $4(4-5)$ & 0.38 \\
\hline
\end{tabular}

All values stated as medians (interquartile range), unless otherwise stated.

a Junior versus senior: intervention arm, $P=0.209$; control arm, $P=0.804$.

${ }^{\mathrm{b}}$ Junior versus senior: intervention arm, $P=0.176$; control arm, $P=0.828$.

' Junior versus senior: intervention arm, $P=0.221$; control arm, $P=0.021$. 
virtual reality is also an added advantage to provide ever-changing tasks to maintain learners' engagement and confer unpredictable new challenges and knowledge. Apart from teaching, the virtual reality scenarios and simulations can be used for competency assessment, which in reality, would have only been possible opportunistically.

While most ED physicians are well equipped with emergency airway management skills as demonstrated by high first-pass intubation success rates in multicentre studies ${ }^{14}$ there is still a proportion of patients with difficult airway features that poses challenges and difficulties in securing the airway. In such patients, awake FBI intubation is the method of choice. ${ }^{15,16}$ However, emergency physicians are often not confident and may not be competent in performing awake FBI intubation at the onset. A 10-year study done by Hayden et al. reports a dismal first-pass success rate of $51.1 \%$ among patients who underwent primary FBI intubations in the ED. ${ }^{17}$ First-pass success in intubations is particularly important and repeated attempts have been found to be associated with increasing peri-intubation adverse events. ${ }^{18-20}$ Hence, there is a need for ED physicians and residents to be well trained and confident in using FBI to manage difficult airway.

In lieu of the above, our study aims to evaluate whether the use of virtual reality via a gaming app can enhance the training of FBI, and we found an improvement of 11 seconds in time to successful intubation among the junior physicians in the intervention group. Although this result did not reach statistical significance, the additional time saved has clinical importance in critically ill patients who may desaturate rapidly to critical levels of oxygen saturation of less than $70 \%$ within seconds. ${ }^{21}$ On the other hand, the intervention did not seem to improve the time taken to successful intubation among the senior physicians. Although the senior physicians in the intervention group did perform slightly better than the control group-respectively, 8.5 seconds versus 12 seconds in time to first vocal cords visualisation $(P=0.23)$ and 45.5 seconds versus 48 seconds in time to successful endotracheal tube placement $(P=0.87)$ - this improvement in time from 2.5 to 3.5 seconds (statistically insignificant) is unlikely to be clinically significant. Additionally, scope manipulation skills were significantly better among junior physicians in the intervention group compared to the control group but this difference was not observed among the senior physicians between the 2 arms. Even though there were higher scope manipulation ratings in the senior control group than the junior control group, we speculate that this may be due to prior bronchoscopic experience among some senior participants, though the study was not designed to specifically address the reasons for this result. Nevertheless, integrating virtual reality technology with a mobile gaming app in FBI teaching appeared to have enhanced the quality of skill acquired, particularly among the junior physicians. We postulate several reasons for this.

A few concepts that underpin simulation-based learning have been described, some of which comprise the acquisition of technical proficiency that includes psychomotor skills, learning theory and the effect of emotion on learning. ${ }^{22}$ It is possible that the senior physicians may have a lower ability to acquire psychomotor skills through virtual reality. Moreover, senior physicians are likely to have preconceived ideas on how a procedure needs to be done based on their exposure and experience, triggering a need to unlearn and learn, which can have an impact on emotions and ability to absorb new learning experience. Another postulation involves seniority and increased experience where advanced learners may feel an inadequacy of realism when learning through virtual reality, leading to inability to immerse. Simulation technology requires the learner to fully submerge in the experience ${ }^{23}$ for greater benefits and educational gains. Despite extensive research on the effectiveness of simulation teaching in medical education, little has been studied on its efficacy among learners of different age groups. This should be explored further in future studies.

\section{Limitations}

This study has some limitations. First, we were unable to demonstrate how skills and confidence gained through such learning could be practicable in clinical practice and patient outcomes, since clinical encounters present opportunistically. Second, we did not measure the baseline timings before training, which could be a possible confounder to our results. Third, there was an oversimplistic representation of the airway anatomy in the lowfidelity manikin used in our study. However, assessment in live patients would have been ethically controversial and may have subjected each participant to anatomical differences. Also, there would be limited number of patients requiring FBI leading to an inability to assess participants on the same day or at all.

Fourth, our study design involved partial immersion instead of full immersion, and the latter may achieve better educational gains for learners. ${ }^{24}$ In relation to this, we chose a mobile gaming app that incorporates virtual reality instead of a virtual reality simulator that may facilitate a higher level of immersion given that a gaming app can be easily assessed from mobile devices, and without purchase of additional hardware. We felt that 
this could provide learners an opportunity for selfdirected and flexible learning, anytime and anywhere, which would not be possible with a simulator. Fifth, other aspects of clinical performance were not assessed, such as patient positioning, jaw or tongue control, management of secretions and passage of a tracheal tube over the scope. Additionally, non-technical skills such as task management, communications and decision-making were also not addressed. Finally, skill retention or decay over time were not within the scope of evaluation in our study.

\section{CONCLUSION}

FBI remains a rare but important procedure to master for emergency physicians. Training using virtual reality, together with traditional teaching methods allow learners to be trained on this life-saving skill in a safe environment without compromising patient care. Junior physicians appear to benefit more from such training compared to senior physicians.

\section{Acknowledgements}

We thank our colleagues in the Emergency Medicine Department of National University Hospital, Singapore, for their participation and support in this research study.

\section{REFERENCES}

1. Ezri T, Szmuk P, Warters RD, et al. Difficult airway management practice patterns among anesthesiologists practicing in the United States: Have we made any progress? J Clin Anesth 2003;15:418-22.

2. Mlinek EJ Jr, Clinton JE, Plummer D, et al. Fiberoptic intubation in the emergency department. Ann Emerg Med 1990;9:359-62.

3. Weiss YG, Deutschman CS. The role of fibreoptic bronchoscopy in airway management of the critically ill patient. Crit Care Clin 2000;16:445-51

4. Marsland C, Larsen P, Segal R, et al. Proficient manipulation of fibreoptic bronchoscope to carina by novices on first clinical attempt after specialized bench practice. Br J Anaesth 2010;104:375-81.

5. Naik VN, Matsumoto ED, Houston PL, et al. Fiberoptic Orotracheal Intubation on Anesthetized Patients. Anesthesiology 2001;95:343-8.

6. Bray JK, Yentis SM. Attitudes of patients and anaesthetists to informed consent for specialist airway techniques. Anaesthesia 2002;57:1012-5.

7. Ovassapian A, Dykes MHM, Golmon ME. A training programme for fibreoptic nasotracheal intubation. Use of model and live patients. Anaesthesia 1983;38:795-8.
8. Schebesta K, Hüpfl M, Rössler B, et al. Degrees of reality: airway anatomy of high-fidelity human patient simulators and airway trainers. Anesthesiology 2012;116:1204-9.

9. De Oliveira GS, Glassenberg R, Chang R, et al. Virtual airway simulation to improve dexterity among novices performing fibreoptic intubation. Anaesthesia 2013;68:1053-8.

10. Farmery AD, Roe PG. A model to describe the rate of oxyhaemoglobin desaturation during apnoea. Br J Anaesth 1996;76:284-91.

11. Bowen-Loftin R. Virtual environments for aerospace training. In: Proceedings of WESCON/94, Idea/Microelectronics. New York: IEEE; 1994, pp. 384-7.

12. Gorman PJ, Meier AH, Krummel TM. Simulators and virtual reality in surgical education. Arch Surg 1999;134:1203-8.

13. Scalese RJ, Obeso VT, Issenberg SB. Simulation technology for skills training and competency assessment in medical education. J Gen Intern Med 2008;23:46-9.

14. Park L, Zeng I, Brainard A. Systematic review and meta-analysis of first-pass success rates in emergency department intubation: Creating a benchmark for emergency airway care. Emerg Med Australas 2017;29:40-7.

15. Malhotra S. Practice Guidelines for Management of the Difficult Airway. Pract Guidel Anesth 2016;5:127.

16. Frerk C, Mitchell VS, McNarry AF, et al. Difficult Airway Society 2015 guidelines for management of unanticipated difficult intubation in adults. Br J Anaesth 2015;115:827-48.

17. Hayden EM, Pallin DJ, Wilcox SR, et al. Emergency department adult fiberoptic intubations: incidence, indications, and implications for training. Acad Emerg Med 2018;25:1263-7.

18. Sakles JC, Chiu S, Mosier J, et al. The importance of first pass success when performing orotracheal intubation in the emergency department. Acad Emerg Med 2013;20:71-8.

19. Hasegawa K, Shigemitsu K, Hagiwara Y, et al. Association between repeated intubation attempts and adverse events in emergency departments: an analysis of a multicenter prospective observational study. Ann Emerg Med 2012;60:749-54.

20. Mort TC. Emergency tracheal intubation: complications associated with repeated laryngoscopic attempts. Anesth Analg 2004;99:607-13.

21. Weingart SD, Levitan RM. Preoxygenation and prevention of desaturation during emergency airway management. Ann Emerg Med 2012;59:165-75.

22. Kneebone R. Evaluating clinical simulations for learning procedural skills: A theory-based approach. Acad Med 2005;80:549-53.

23. Carroll JD, Messenger JD. Medical simulation: the new tool for training and skill assessment. Perspect Biol Med 2008;51:47-60.

24. Gutiérrez F, Pierce J, Vergara VM, et al. The effect of degree of immersion upon learning performance in virtual reality simulations for medical education. In: Al JDW et (Ed) Medicine meets virtual reality. Fairfax: IOS Press; 2007, pp. 155-60. 